\title{
The Application Mode in Urban Transportation Management Based on Internet of Things
}

\author{
Linna Huang \\ Department of Computer Engineering \\ CangZhou Normal University \\ Cangzhou, China \\ Hln0322@163.com
}

\author{
Chunli Liu \\ Department of Computer Engineering \\ CangZhou Normal University \\ Cangzhou, China \\ Chunliwl@163.com
}

\begin{abstract}
The concept of intelligent transportation has been accepted by the community and has also been widely used. Meanwhile, the problems of intelligent transportation systems have revealed. Then, the internet of things provides a new way for the development of intelligent transportation. This paper firstly introduces the concepts, principles and structure of internet of things. Then, it analyses the impact on transportation given by internet of things, states the relationship between internet of things and the intelligent transportation and shows the research on the application mode of urban transportation management based on internet of things.
\end{abstract}

Keywords- intelligent transportation; internet of things; intelligent; RFID

\section{INTRODUCTION TO IOT TECHNOLOGY}

IoT (internet of things) is called the second computer. After the outcome of internet, this is the third impact in the world information industry. IoT is based on RFID (radio frequency identification), infrared sensors, global positioning systems, laser scanners and other information sensing devices. According to the agreed protocol, the network is related to any articles connected with the internet, information exchange and communication in order to achieve intelligent identify, location, track, monitor and management. As the name suggests IoT is "a network of Internet-enabled objects." It has two meanings: First, the core and foundation of IoT are still the Internet and it is the extension and expansion of the network. Second, the clientend extends to any goods and objects based on the exchange of information and communication, which is composed of many sensors. [1] So IoT is an Internet-based, traditional telecommunications networks and other information carrier, so that all can be addressed independently for ordinary physical objects to achieve interoperability. Specifically speaking, the bridges, tunnels, roads and other structures are embedded and quipped with sensors in the transportation and they are generally connected to each other. So it is called IoT.

\section{INTELLIGENT TRANSPORTATION SYSTEMS}

ITS (Intelligent Transportation Systems) refers to the advanced sensor technology, information technology, network technology, automatic control technology, computer processing technology used in the transportation management system to form a kind of information, intelligence, socialization transport integrated management and control systems. Intelligent transportation system makes transport infrastructure to maximize performance. The technology originated in the 1980s in the United States and other countries are actively pursuing the development in this area. With the Internet, movement of communication, sensor networks and other new forms of technology. IoT have taken shape in intelligent transportation and will have a strong potential for development in the next few years [2].

\section{IOT AND INTELLIGENT TRANSPORTATION}

Transportation IoT is put forward in the context of the strategic initiative of IoT. Based on transportation IoT, the whole transportation can be tracked to ensure transport safety; intelligent urban transportation management and achieve much more transportation information of vehicle, automatic driving implementation and so on.

Actually, in real life we can see specific applications of transportation IoT, such as non-stop electronic highway toll, intelligent transportation systems, mobile emergency command and scheduling, transportation police mobile law enforcement, vehicle transportation violation monitoring, eport, vehicle anti-theft system etc. But these are just the beginning and are not yet a huge network. In the future, we can imagine the connections between vehicles and vehicles, between people and vehicles and between vehicles and roads as a huge intelligent transportation so as to solve a lot of transportation congestion, environmental pollution and traffic security.

\section{A. The core of intelligent transportation is collaboration}

of people, vehicles, and roads

Intelligent Transportation is a balanced application of science. In order to balance the transport users, vehicles and limited roads, we can find out the time of the arrival of next bus, availability of empty seats, the best route and avoidance of congested roads. You can sense the vehicle brake in front of you in the shortest time and avoid the accident by taking measures in the future transport system.

Therefore, the first important step of intelligent transportation system is to achieve the data. Through the placement of sensors everywhere, transportation managements can access real-time transportation information to help monitor and control transportation flow. Through the installation of GPS in cars and radio frequency tags, the traffic police can exchange surrounding traffic information at 
any time to obtain valid transportation information to guide vehicles to change or optimize travel routes. In a sense, intelligent transportation is the application of IoT technology in transportation fields.

To develop people, vehicles, and roads collaboratively is the core of intelligent transportation systems [3], which is just a perfect interpretation of the concept of transportation IoT. The proposed transportation IoT has greatly promoted the development of intelligent transportation. For example, transportation IoT awareness, network, platform, application of the four level have been introduced in the architecture design system of Shenzhen intelligent transportation systems in the context of transportation IoT so as to achieve innovative design of "intelligent transportation " in the era of transportation IoT.

\section{B. Related technologies and applications}

The core of Iot is the information data collection and processing. The key to transport vehicle networking technology is how to achieve information exchange between vehicles and roads and between vehicles and vehicles. Undoubtedly wireless technology plays a major role.

Current vehicle location, communications and tolls are more based on the application of DSRC (short-range communications technology) and VPS (vehicle positioning system) technology. DSRC is a microwave technology, mainly used in the electronic road pricing system. The VPS is GPS + GSM technology. It is more widely used in car navigation and voice communication. There is no need to discuss it because these technologies are existing technologies.

In addition, infrared and ultrasonic technology is also widely used, which is simple and environmentally friendly technologies. The other core technologies of transportation IoT include radio frequency identification devices, video detectors, magnetic sensors, wireless sensors, global positioning systems, Internet and wireless communications and industrial application software.

Among these technologies, the most critical one is to develop the underlying chip embedded devices, which leads the entire industry development. Based on the integration of IoT and embedded systems, existing products will be upgraded to make services more intelligent so as to engage remote device management, scheduling and maintenance.

Software is the core and key of IoT application. The major software companies have invested heavily in related software research and design and middleware (SaaS, SOA, cloud computing, etc.). The proposed concept of cloud computing will play a major role in transportation IoT. Transportation is a complex science because of uncertainty of each transport objects. Cloud computing as a highly scalable method of calculation provides users with resources in form of "on-demand service" via Internet and meets the complex transportation requirements "use on demand, charges for use and no use after completion." [4-5]

From a technical level, the key technologies to develop transportation IoT include: (1) DSRC-short-range communications technology (2) the vehicle running state detection technology (3) infrastructure and environmental performance detection technology (4) driver assistance technologies (5) a new generation of transportation control system.

\section{ANALYSIS ON APPLICATION MODE OF IOT TECHNOLOGY IN URBAN TRAFFIC MANAGEMENT}

\section{A. RFID-based electronic license plate system functions}

RFID (Radio Frequency Identification) commonly known as E-tag is a non-contact automatic identification technology, which achieves the relevant data through RF signal automatic target recognition. It can work without human intervention in harsh environment. RFID technology can identify fast moving objects and multiple tags quickly and easily. RFID is a simple wireless system with only two basic components. The system is used to control, detect and track objects. System consists of an interrogator (or reader) and a lot of transponders (or tags). It utilizes frequency RF to have non-contact transmission of data in two ways between the reader and RF card in order to achieve target identification and data exchange.

The main functions of RFID-based electronic license plate system in the urban transportation management are (1) Vehicle license information management. The license plate information corresponds to the vehicle. For instance, the valid date of the plate can be obtained from electronic license plate on the road at any time (2) Mobile / specific road vehicle inspection. Normally traffic police inspect vehicles for traffic rules and regulation enforcement to ensure safe and orderly transportation on the road with vehicles lawfully driven, no overspeed and no stopping on the road. (3) Non stop toll collection. It is mainly used in the provincial and highway toll to ensure rapid payment of vehicles. (4) Automatic car park management including parking and access statistics. (5) The statistical transportation data and vehicle specific information management for illegal operation and security. It provides accurate transportation flow data of urban roads, highways, and an accurate basis for the transportation planning in order to have the smooth and orderly transportation. (6) Drunk driving monitoring and management. It primarily detect whether the driver is driving unusually. If it is drunk driving, the car will be recorded after the confirmation and the vehicle will be monitored afterwards if on the road.

\section{B. Function Implementation of RFID-based electronic license plate system}

\section{1) License information management}

License information can be successfully obtained through the radio frequency identification devices at the road junctions and for information database of license plate information center when the vehicle is passing. The vehicle will be automatically prompted to take the necessary measures if the license has expired or the vehicle fails to do the annual car inspection. Electronic license plate can also provide services for the vehicle inspection with no need to be at a fixed location. The driver can do it at the small and large frequency identification terminal in the cities. When the vehicle passes at the terminals, the IC card of the car can be 
automatically updated, and recorded in the database. Thus it is more efficient to complete inspection work and saves a lot of time for the drivers and as well as a lot of manpower and resources for managements.

\section{2) Monitoring illegal driving and vehicles involved}

Transportation management can not only monitor transportation on the main road through the fixed radio frequency identification devices but also actively implement the vehicles safety driving management on the road by using mobile RFID terminal with dynamic monitoring of vehicles on certain roads, especially for the overspeed vehicles in order to take the appropriate measures to ensure the orderly driving. The radio frequency identification RFID technology is used in the key management and services for dangerous goods and freight vehicles and also security pass and vehicle access control limit, etc. Radio frequency identification devices are installed at the important exits and entrances to spot dangerous goods vehicles and illegally driven cars through a certain frequency band identify and control their access, which is much quicker and more efficient than manual detection.

Due to the need for vehicle control in and around the Expo, The application of car driving detection system played a great role during the Shanghai Expo. The roads around the Expo were appointed as transportation control area. RFID was installed in the key control area to monitor vehicles around the Expo for 24 hours and transmit the information back to the Expo transportation control centers. If any unusual circumstances, the center can spot first at once and send transportation control command to traffic police at the various locations to take the relevant transportation management measures. Meanwhile, automatic alarm system was on for detecting suspect license plates. Suspect vehicles will immediately advise to stop and perform relevant measures if license plates do not meet the requirements.

3) Toll collection without car stopping

Toll collection without car stopping happens mainly at inter-provincial highway and expressway tolls. Charges and fees will be deducted through the automated radio frequency identification technology by automatically scanning IC cards of the passing vehicles and of course, IC card management services are also provided, such as, IC card processing, cancellation, replacement, and recharge. This will greatly improve the efficiency of toll services to ensure vehicle nonstop while the size of the toll itself can be greatly reduced so as to save money and resources.

4) Automatic parking management

On automatic parking management functionality, the application of intelligent electronic license plates has stated in details in the above transportation management. So the statements will not be repeated here.

5) Monitoring of transportation flow

The flow data obtained from monitoring of transportation is the important basis for traffic control police and managements to make a decision. In addition to radio frequency identification technology, the sources of transportation data are also obtained through GPS (Global Positioning System), GIS (geographic information system) and other ways of data collection. Using global positioning system is to achieve the dynamic positioning of the vehicle tracking and the necessary information about certain vehicles. The use of geographic information system is mainly to obtain information about fixed sections of roads, such as, numbering the fixed peak sections, road network data and information of transportation flow. Sometimes timely access to transportation flow data is also to monitor transportation, detect illegal transportation behavior and deal with traffic accidents for the first time. The common installation of monitoring devices is gantry type.

To make efficient transportation decision-making is bound to have a high standard for data: accuracy, a large amount and timely updates. Here, modern network communication technology has contributed a lot. With the large number of advanced GSM network running, access to transportation data can be transmitted at a great speed between the transport networks. Moreover, the quality can be guaranteed with accurate data transmission and there is no limit for the transmission. In the information center, it is possible to use database technology to maintain a large amount of database. Computers can receive a large amount of data in time to classify and store it. Data can be searched and obtained when needed. The data is also updated at a fixed time because it is related to accuracy which transportation managements will base on and also it is significant for transportation decision makers to make a decision.

A large amount of data can be used for the simulation in the information center, depending on the cloud computing technology so that scientific and reasonable conclusions will be presumed. These findings are important information for decision-makers to base on. Meanwhile it is also important for drivers to refer to the transportation information, for instance, routing selection. Drivers can select the most suitable routes according to real-time information of surrounding transportation flow and history transportation flow. Today, the availability of new means of communication provides an accessible tool to put the functions into practice.

6) Management after alcohol drinking

It is well known that it is dangerous to drive after drinking alcohol. But it is quite difficult to deal with it. Radio frequency identification technology is used to monitor the car driving on the roads. If the driving is found not steady or parallel to the road, the car will be tacked and monitored by using image analysis techniques to further determine whether the car is drunk driving and contact the device in the car through the exchange of real-time information service and finally instruct police patrol to have alcohol testing.

\section{CONCLUSION}

From the above we can see if we want to achieve the various functions of intelligent transportation, one technology is far from enough and we must use a variety of IoT technologies especially global positioning technology, geographic information systems technology and comprehensive use of radio frequency identification 
technology for urban transport management so as to bring a qualitative leap.

With the development of IoT technology, there will be more and better applications of intelligent transportation based on IoT. It has great significance in improvement of transportation management quality and the level of management. First, more thorough perception. Sensor and vehicle sensing devices are used to monitor transportation flow with more real-time vehicle status and the information will be sent to the network management center. Second, more comprehensive interoperability. Road environmental status will be provided to people on travelling so as to optimize people's travelling. Third, more in-depth intelligence. Road infrastructure will be greatly utilized through the management and scheduling mechanisms so as to maximize network transportation and improve transportation safety.

Assume that we use IoT technology to achieve the goal, i.e. optimized application prospects: People know in advance to avoid the transportation congestion and drive along the fastest route to reach destinations so as to reduce fuel consumption and carbon dioxide residual emissions. People on travelling can obtain timely weather information. Drivers can always find the nearest parking space and even they can use auto pilot to ensure driver's relaxation. Passengers can enjoy high-quality online entertainment services on their journey.

IoT has good prospects, but after all it is just a new technology to develop from the Internet. It must be combined with existing industry to develop products to bring the commercial value. It can be well integrated into intelligent transportation field due to IoT technical characteristics. With the further development of IoT technology and the construction of intelligent transportation in big cities, a wide variety of industrial applications will be put into practice in intelligent transportation and urban intelligent transportation will bring a qualitative leap. Electronic license plate as an example of the integration of radio frequency identification technology, global positioning technology, geographic information systems etc is one of the products used IoT technology, which will play a huge role in urban transportation management in the future.

\section{REFERENCES}

[1] Li Ye, Wang Jingbo, Dong Libo, "The Research on the application of Intelligent Transportation Based on IoT," Mobile Communication, No.15, pp. 30-34, 2010

[2] Chen YuFeng, Xiang ZhengTao, Chen Li, "The Research Development on the Transportation Information collection technology of Intelligent Transportation system,” Hubei Automobile Industry Institute Journal, Vol. 24, No.2, pp. 30-36, 2010.

[3] Ye Jiasheng, "Road Traffic Information Collection Based on FCD Technology and Dynamic Transportation Guidance System,” Hefei University master's degree thesis, 2009.

[4] Guo Jun, "RFID technology Application in Urban Road Transportation Management," Transportation Construction and Management, pp. 84-86, August 2009.

[5] Min Je Cho, Hyung Rim Choi, Byung-Kwon Park, Kangbae Lee, Soon Goo Hong, Chang Hyun Park, Gwang Rok Kim, "A Disaster Prevention System in the Transportation of Dangerous Goods by Using IP-RFID”, JDCTA, Vol. 6, No. 1, pp. 384-390, 2012. 